\title{
FORMATION AND EVOLUTION OF HIERARCHICAL SYSTEMS
}

\author{
Sverre J. Aarseth ${ }^{1,2}$ \\ RESUMEN
}

Se utilizan distribuciones iniciales de binarias primordiales en simulaciones realistas de $N$-cuerpos que dan origen a subsistemas ligados de diferente multiplicidad. Un examen del proceso de formación muestra que las triples más compactas se originan en colisiones binaria-binaria, mientras que los sistemas de orden mayor tienen orígenes diversos. Las tasas de formación son bajas, pero se compensan con vidas medias largas, lo cual lleva a un aumento significativo de la población de sistemas múltiples. Al ser bastante energética, la órbita exterior tiende a contraerse en los encuentros subsecuentes. Además, las perturbaciones externas también modifican la excentricidad, y pueden crear condiciones propicias para la inestabilidad. Un resultado característico es la destrucción interna por el efecto honda. Estas interacciones son con frecuencia suficientemente energéticas como para producir escapes a alta velocidad, y no es raro que algunas dobles o triples salgan expelidas. Para inclinaciones altas, el crecimiento de la excentricidad inducido por el mecanismo de Kozai puede llevar a una contracción apreciable de la órbita de la binaria interior, a causa de la circularización por efecto de marea. $\mathrm{Si}$ algunas de las componentes se fisionan para formar binarias ultracerradas, dinámicamente inactivas, las jerarquías formarían multiplicidades de aún mayor orden. Estos resultados implican que una parte de las múltiples de campo observadas pudieron haberse formado en un ambiente dinámico.

\section{ABSTRACT}

Initial distributions of primordial binaries used in realistic $N$-body simulations give rise to bound subsystems of different multiplicity. An examination of the formation process shows that the most compact triples arise via binary-binary collisions, whereas higher-order systems have diverse origins. Low formation rates are compensated by long life-times, leading to a significant population building up. Being fairly energetic, the outer orbit tends to shrink by further encounters. In addition, external perturbations also modify the eccentricity and may create conditions for instability. One characteristic outcome is internal disruption by the sling-shot mechanism. Such interactions are often sufficiently energetic to produce high-velocity escapers and it is not uncommon for triples and quadruples to be ejected. For high inclinations, the eccentricity growth induced by the Kozai effect may lead to significant shrinkage of the inner binary orbit by tidal circularization. If some of the components were spit into a dynamically inactive ultra-hard binary, the hierarchies would be attributed an even higher multiplicity. One implication of these results is that a proportion of multiple systems observed in the field may have been formed in a dynamical environment.

\section{Key Words: BINARIES: GENERAL - METHODS: N-BODY SIMULATIONS - OPEN CLUSTERS AND ASSOCIATIONS}

\section{INTRODUCTION}

Given that star clusters often reveal a large fraction of binaries with a wide range of separations, it is natural to suppose that multiple subsystems should also be present. Observational evidence for triples in clusters is now beginning to accumulate (Raboud \& Mermilliod, 1998) and a first analysis of triples in the field has been made (Sterzik \& Tokovinin, 2002). At the same time, direct $N$-body simulations of rich open clusters containing primordial binaries show that long-lived multiple systems formed in situ play an important role (Aarseth, 2001). In fact, their

\footnotetext{
${ }^{1}$ Institute of Astronomy, University of Cambridge, UK.

${ }^{2}$ Madingley Road, Cambridge CB3 0HA, UK.
}

very presence poses a considerable challenge for the numerical analyst since the inner binary often has a small period. Moreover, subsequent strong interactions between multiple systems and single stars or binaries may produce high-velocity escapers with interesting observational consequences. A variety of tools are brought to bear on trying to understand the formation and evolution of such systems but this study is still in the early stages.

In this paper, we examine some ideas about different formation mechanisms and consider various aspects of the subsequent evolution. Since the discussion is based on data from numerical simulations, we begin by describing the essential features of a 
typical cluster model. The question of how to identify a newly formed subsystem is not an easy one to implement and requires special considerations.

\section{CLUSTER MODELS}

For the present purpose, we define a standard model of an open cluster as consisting of $N_{\mathrm{s}}=8000$ single stars and $N_{\mathrm{b}}=2000$ primordial binaries, making a total of 12000 stars. We adopt a conventional power-law IMF in the range $15-0.2 M_{\odot}$ and the binaries having periods between 4 and $10^{6}$ days with the component masses selected independently. In addition to the internal interactions, we include an external tidal field characteristic of a circular orbit in the Solar neighbourhood with a corresponding tidal radius of about $28 \mathrm{pc}$. This compares with a typical half-mass radius of $r_{\mathrm{h}} \simeq 4 \mathrm{pc}$ which defines a standard crossing time of about $3.5 \mathrm{Myr}$. Some results will also be shown from a second similar model with $N_{\mathrm{s}}=9000$ and $N_{\mathrm{b}}=9000$ to explore the importance of a large binary fraction.

In order to perform realistic cluster simulations we include all the main processes of stellar evolution by means of a synthetic description involving fast fitting functions (Tout et al., 1997). Thus not only slow and fast mass loss are modelled but also tidal circularization (Mardling \& Aarseth, 2001), Rochelobe mass transfer and physical collisions. A special averaging treatment of highly inclined inner binaries in triples due eccentricity modulation (Kozai, 1962) is also included (Aarseth \& Mardling, 2001).

The equations of motion for the single particles are advanced by the fourth-order Hermite integration method (Makino, 1991) which has proved to be efficient on a variety of hardware. The calculations of model 1 were performed on the HARP-2 special-purpose computer at the Institute, whereas the more powerful GRAPE-6 was used to study the larger model 2 which requires a considerably greater effort. All hard binaries and close encounters are treated by two-body regularization (Kustaanheimo \& Stiefel, 1965), whereas strong interactions involving 3-5 members are studied by chain regularization (Mikkola \& Aarseth, 1993). A summary of all the relevant methods has been given elsewhere (Aarseth, 1999). As a convenient definition of a close encounter, an expression of the form $R_{\mathrm{cl}} \simeq r_{h} / N$ is adopted and this also applies approximately to the size of hard binaries of average mass.

The overall evolution of the cluster is characterized by a reduction of the membership due to escape. Hence the tidal radius tends to shrink slowly, while the half-mass radius is fairly constant during most of

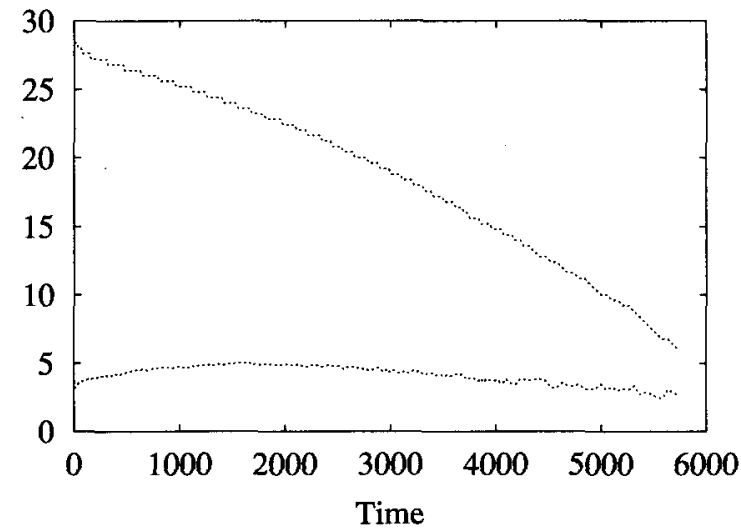

Fig. 1. Tidal radius and half-mass radius in pc as function of time in Myr for model 1.

the evolution. These quantities are shown in Fig. 1. We remark that the core radius is about one-fifth of the half-mass radius.

\section{FORMATION OF TRIPLES}

The data structure employed is convenient for identifying processes leading to the formation and evolution of long-lived multiple systems which are invariably connected with binaries. The main mechanism for the formation of triples was elucidated in binary-binary scattering experiments a long time ago (Mikkola, 1983) but this was not exploited until quite recently. In fact, the process can be understood as the hardest binary being exchanged with one of the components of the second pair which is ejected. Since the binaries tend to be concentrated towards the cluster centre, a relatively modest initial population may be sufficient to produce a significant number of bound triples (Aarseth, 2001). Strong binary-binary interactions give rise to two different outcomes involving destruction of one of the binaries. This can be written in symbolic form as

$$
\begin{aligned}
& B+B=B^{\prime}+S+S \\
& B+B=T+S,
\end{aligned}
$$

with $T$ denoting a bound triple and S a single star. Thus the first case includes the possibility of the remaining binary undergoing exchange.

Given the presence of a bound triple system, the question of its stability naturally arises. A simple approach would be to monitor the system during the subsequent time until possible disruption or ejection. However, the numerical treatment itself can be speeded up considerably while retaining a consistent description once a stability test has been accepted. 


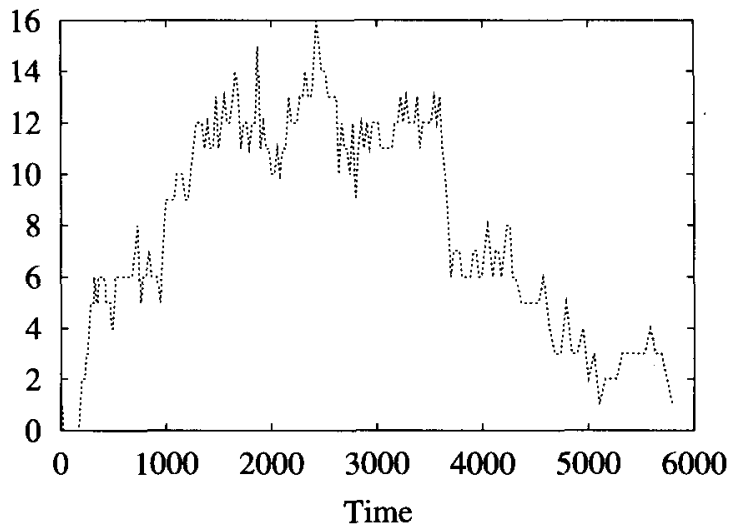

Fig. 2. The number of stable hierarchical systems in model 1 .

An implementation of this procedure is greatly facilitated by the use of regularization for dominant two-body motion.

The standard case of a stable triple can be considered as two binaries, with the outer orbit well separated from the inner one, and such a configuration is best described by hierarchical coordinates. A semi-analytical stability criterion in terms of the masses and orbital elements is available, replacing earlier more approximate expressions (Harrington, 1972; Eggleton \& Kiseleva, 1995). It is based on a correspondence between an oscillating star in the binary tides problem and the inner binary of a triple (Mardling, 1995). The boundary value of the outer pericentre distance for planar prograde motion is given by (Mardling \& Aarseth, 1999)

$$
R_{p}^{\text {crit }}=C\left[\left(1+q_{\text {out }}\right) \frac{\left(1+e_{\text {out }}\right)}{\left(1-e_{\text {out }}\right)^{1 / 2}}\right]^{2 / 5} a_{\text {in }},
$$

where $q_{\text {out }}=m_{3} /\left(m_{1}+m_{2}\right)$ is the mass ratio of the outer body with eccentricity $e_{\text {out }}$ and $a_{\text {in }}$ is the inner semi-major axis. The constant $C \simeq 2.8$ is determined empirically at present. A small heuristic correction of up to $30 \%$ is then applied for non-inclined orbits to account for the increased stability. Extensive numerical tests of three-body systems have demonstrated that the criterion is robust for a wide range of parameters but so far planetary masses are excluded. It should be emphasized that this derivation applies to escape of the outer body. However, the alternative case of exchange, which is governed by the Zare (1977) criterion, is only more stringent for $q_{\text {out }} \geq 5$ and hence of less practical relevance here.

Provided that we have $a_{\text {out }}\left(1-e_{\text {out }}\right)>\tilde{R}_{\text {crit }}$, the system is defined to be stable in the absence of further external perturbations and significant mass loss. By adopting stability according to this definition, we assume that the inner semi-major axis is secularly constant and neglect short-term fluctuations. This property has been verified in accurate integrations using three-body regularization (Aarseth $\&$ Zare, 1974). Consequently, the inner binary can be replaced by the combined mass, which allows the outer orbit to be treated as a regularized two-body solution. In order to ensure significant life-times, only configurations satisfying the hard energy condition within a factor of 0.25 for the outer orbit are accepted for special treatment. We defer a discussion of the so-called 'Kozai effect' until a later section.

Figure 2 shows the population of stable systems in model 1. In the absence of primordial hierarchies, there is a gradual increase with about 12 members maintained over at least $2 \mathrm{Gyr}$. Although this is a small fraction of the total number of binaries, many of these systems are important dynamically by virtue of their central concentration, larger mass and increased cross section.

An estimate of the typical life-times may be made from the available data. First we record any stable triple formed during chain regularization which only treats the strongest interactions and hence represents the most extreme cases. There were 14 such examples out of about 630 chain treatments. Each new configuration accepted as stable is also recorded, taking care not to include recent configurations which have been terminated temporarily due to large perturbations without changing the membership. If we assume that the 300 such events represents an upper limit, the typical life-time would correspond to about $100 \mathrm{Myr}$. Hence we may conclude that the long life-time implies a small formation rate.

As expected, the richer cluster model produced a substantially greater number of hierarchical systems. Figure 3 shows that a characteristic membership of at least 30 stable systems was maintained over some $3 \mathrm{Gyr}$, with a maximum of about 50 . It should be emphasized that all these systems satisfy the stability criterion as well as the hard binary condition to within a factor of 0.25 . Hence the total number of hierarchies at a given time will undoubtedly be somewhat larger.

\section{EVOLUTION OF HIERARCHIES}

Once formed, a stable hierarchy tends to evolve in much the same way as a hard binary, with exchange of the outer component being one way of increasing the total mass and energy. However, there are also 


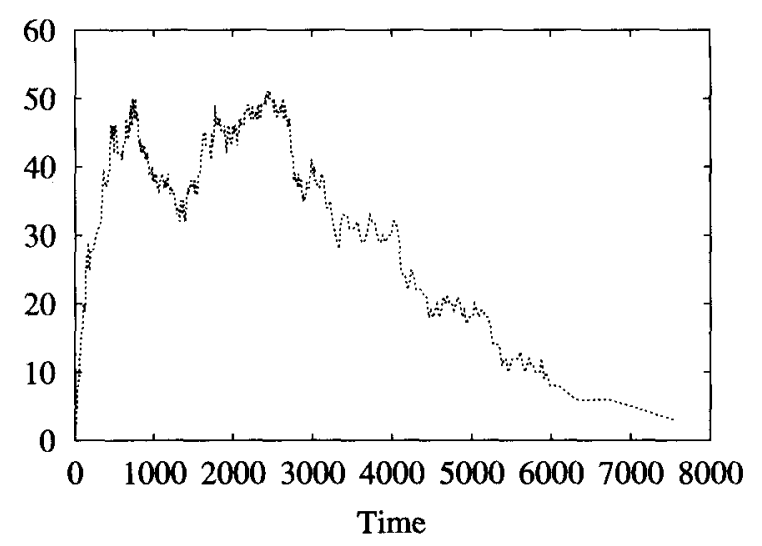

Fig. 3. The number of stable hierarchical systems in model 2.

additional channels of outcome which may lead to internal disruption. This can occur after sufficient shrinkage of the outer orbit until the system becomes unstable to exchange (Zare, 1977). Alternatively, the outer eccentricity may undergo small changes due to systematic perturbations, giving rise to the slingshot condition

$$
a_{\text {out }}\left(1-e_{\text {out }}\right) \simeq a_{\text {in }}\left(1+e_{\text {in }}\right),
$$

where high recoil velocities are possible (Saslaw, Valtonen \& Aarseth, 1974). Thus it is known from theoretical considerations that the eccentricity change of a binary depends on the perturbation to first order whereas the semi-major axis change is to second order (Heggie, 1975).

In addition to internal destruction by dynamical means or mass loss from evolving stars, bound triples may also be ejected from the cluster. In model 1 , some 13 triples escaped mostly with significant excess velocity which would indicate energetic interactions. It should be noted that these ejections only occur after about $2.6 \mathrm{Gyr}$ when the central cluster potential has been reduced in strength by a factor of 3. Likewise, there were 40 ejected triples in model 2 , typically with significant terminal velocity. Analysis of the sample of escaped triples shows a preference for large masses, with average values of 3.0 and $2.2 M_{\odot}$, compared to $0.5 M_{\odot}$ for single stars. This result merely reflects the higher formation probability of massive systems.

One way that a triple may achieve a high recoil velocity is for a quadruple system to suffer internal disruption. However, on the basis of the present small sample, exchange of the outer component is more likely to yield high velocities. For completeness, we also mention examples of ejection due to hardening of the outer orbit as well as encounters where the orbital elements are unchanged. As an example of the latter, we may have an interaction between a triple and a massive binary with minimum separation well in excess of the system size and yet be within the close encounter distance.

The orbital inclination of triples introduces an interesting new feature which can be modelled. Although the inner semi-major axis of stable systems is assumed to be constant, the eccentricity is subject to cyclical oscillations for inclinations in the neighbourhood of $90^{\circ}$. The Kozai (1962) effect is studied by an averaging procedure (Aarseth \& Mardling, 2001) in cases where the tidal circularization time becomes sufficiently short. At large values of the eccentricity we also include friction and general relativistic precession which combine to limit the theoretical maximum value being reached. If still accepted for tidal circularization treatment, the subsequent evolution at constant angular momentum then leads to significant orbital shrinkage and hence enhanced stability. The implication of this process is that a few close binaries may form from wider ones, producing very large period ratios in triples. An observational distinction seems difficult since short-period binaries are also expected to be circularized and the tidal friction is sufficiently effective to prevent eccentricity growth. However, it is expected that recently circularized binaries will be fairly close to the Roche-lobe overflow condition even if the radii have not changed further. Since stellar radii of non-degenerate stars tend to increase with time, certain close binaries may therefore be ruled out as having been circularized.

\section{HIGHER-ORDER SYSTEMS}

If the outer member of a stable triple is replaced by a small binary, general considerations suggest that the configuration may still be stable since the tidal perturbation falls off rapidly with distance. Consequently, we adopt a slightly modified criterion which takes account of the size of a second binary. However, the main channel of triple formation is no longer applicable and different software is needed in order to elucidate this process.

One promising way to identify the early stage of quadruple formation consists of recording the relevant orbital parameters during wide four-body encounters with small impact parameter. This procedure shows many examples of nearly parabolic motion of the two binaries. Consider the total energy of an isolated four-body system consisting of two well separated binaries,

$$
E_{\text {tot }}=E_{\text {out }}+E_{\text {int }}
$$


TABLE 1

A HIERARCHICAL ZOO

\begin{tabular}{ll}
\hline Type & Symbol \\
\hline Classical triple & {$[\mathrm{B}, \mathrm{S}]$} \\
Classical quadruple & {$[\mathrm{B}, \mathrm{B}]$} \\
Quartet & {$[[\mathrm{B}, \mathrm{S}], \mathrm{S}]$} \\
Quintuplet & {$[[\mathrm{B}, \mathrm{S}], \mathrm{B}]$} \\
Quintuplet & {$[[\mathrm{B}, \mathrm{B}], \mathrm{S}]$} \\
Sextuplet & {$[[\mathrm{B}, \mathrm{B}], \mathrm{B}]$} \\
Triplet & {$[[\mathrm{B}, \mathrm{S}],[\mathrm{B}, \mathrm{S}]]$} \\
Septuplet & {$[[\mathrm{B}, \mathrm{B}],[\mathrm{B}, \mathrm{S}]]$} \\
\hline
\end{tabular}

with $E_{\text {int }}$ containing the sum of binary energies. Similarly, we may write an equation for the total angular momentum. Let us now assume that the effect of the external perturbation can be neglected. After the first pericentre passage, some energy may be transferred to the internal motion of the softest pair with a corresponding change of the orbital angular momentum. Since angular momentum increase is favoured for a highly eccentric orbit, the enlarged bjnary will tend to avoid the sling-shot condition at the next passage. This behaviour would then give rise to subsequent shrinkage due to interactions with other stars. If confirmed by more detailed investigations, this interplay between internal and external energy transfer is analogous to the standard process of tidal capture. Thus in order for the centre of mass motion to become bound, the impact parameter needs to be within a small factor of the widest binary size. Other formation mechanisms undoubtedly exist, including three-body encounters, but these contributions are likely to be less important. Although the formation rate of quadruples is very small, such objects are normally present.

Given the occurrence of triples and quadruples in the numerical models, it is natural to expect systems of higher multiplicity. The central density tends to diminish as a result of the general cluster evolution which also includes mass loss due to stellar evolution. This condition gives rise to increased hierarchical space which allows stable configurations of higher multiplicity. In fact, two types of quintuplets as well as standard sextuplets are seen in the simulations, making a veritable zoo of dynamical molecules.

Table 1 lists a number of possible configurations, using a simple symbolic notation and suggestive names. At present the software for identifying complex structures has not been extended to septuplets since there are several types. However, the gen- eralization from triplets can be treated in a similar way and such systems may still be energetically possible for modest eccentricities in evolved clusters. All the other higher-order systems listed here have been identified in large cluster simulations. We make a distinction between quadruples and quartets because different modes of formation are involved. Also note that the two different quintuplet configurations can be considered as having originated from triples and quadruples, respectively. On the basis of this description, quintuplets consisting of an inner quadruple would be quite rare. On the other hand, triplets will tend to be massive which facilitates binary formation near the centre by expelling other particles, leaving behind a bound two-body system (Aarseth, 1972).

Formation histories of high-order systems have been recorded for model 2. Although many are terminated due to large external perturbation, the same configuration often survives over significant time intervals. Thus in one example a quartet remained stable for about $100 \mathrm{Myr}$. In this connection, we mention an observed quadruple system in NGC 2362, albeit with an O-type close binary (van Leeuwen \& van Genderen, 1997).

The cluster models reveal the presence of complex dynamical molecules which enhance the relaxation process of the single stars. Interactions between the diverse components may therefore be discussed in terms of gravitational chemistry. One characteristic outcome of such interactions is the occasional generation of high-velocity escaping stars, with possible terminal values exceeding $\simeq 100 \mathrm{~km} \mathrm{~s}^{-1}$. In this connection, we note that the physical size of the simulated stars places an upper limit on the maximum velocity which can be attained (Leonard, 1991).

\section{CONCLUSIONS}

The present star cluster simulations show that hierarchical systems form naturally if there is a significant fraction of primordial binaries. On the observational side, there is evidence for nearly $100 \%$ binaries in young clusters (Duchêne, 1999) and even $48 \%$ in the Pleiades (Raboud \& Mermilliod, 1998). Since the simulations are time-consuming, only hard binaries are included in the initial distribution and hence a binary fraction of $25 \%$ is a good compromise. In addition, mass segregation and stellar evolution effects lead to preferential central concentration with favourable conditions for triple formation. Once formed, such systems may then combine to form higher-order configurations of increasing complexity, albeit with decreasing life-times. We also 
emphasize that the formation process itself favours massive systems which therefore contribute more effectively to the dynamical evolution.

The analysis of hierarchical systems reveal a fascinating interplay of energy transfer between internal and external modes. Strong interactions are often induced as the result of weak external perturbations, culminating in sling-shot ejections. On the other hand, small internal perturbations produce cyclical oscillations of the inner eccentricity by the Kozai effect and may in certain favourable cases initiate tidal circularization with consequent orbital shrinkage.

A number of multiple systems in the field are known and full orbital parameters have been determined for some (Fekel et al., 2002). As the resolution is increased, more spectroscopic binaries are revealed in clusters (Mathieu, Latham \& Griffin, 1990). Hence the multiplicity of the simulated systems may be even larger if we consider some of the single components as composed of spectroscopic binaries. On the basis of the present $N$-body simulations, we conclude by encouraging observers to look harder at cluster binaries in order to establish higher multiplicities.

\section{REFERENCES}

Aarseth, S. J., 1972, in Gravitational N-body Problem, ed. Lecar, M. (Dordrecht, Reidel), 88

Aarseth, S. J. 1999, PASP, 111, 1333

Aarseth, S. J. 2001, in Dynamics of Star Clusters in the Milky Way, eds. Deiters, S. et al. (San Francisco, ASP), 228, 111.
Aarseth, S. J., \& Mardling, R. A. 2001, in Evolution of Binary and Multiple Star Systems, eds. Podsiadlowski, P. et al. (San Francisco, ASP), 229, 77

Aarseth, S. J., \& Zare, K. 1974, Celest. Mech., 10, 185

Duchêne, G. 1999, A\&A, 341, 547

Eggleton, P. P., \& Kiseleva, L. G. 1995, ApJ, 455, 640

Fekel, F. C., Scarfe, C. D., Barlow, D. J., Hartkopf, W. I., Mason, B., \& McAlister, H. A. 2002, AJ, 123, 1723

Harrington, R. S. 1972, Celest. Mech., 6, 322

Heggie, D. C. 1975 , MNRAS, 173, 729

Kozai, Y. 1962, AJ, 67, 591

Kustaanheimo, P., \& Stiefel, E. 1965, J. Reine Angew. Math., 218, 204

Leonard, P. J. T. 1991, AJ, 101, 562

Makino, J. 1991, ApJ, 369, 200

Mardling, R. A. 1995, ApJ, 450, 722

Mardling, R. A., \& Aarseth, S.J. 1999, in The Dynamics of Small Bodies in the Solar System, eds. Roy, A. E., \& Steves, B. A. (Dordrecht, Kluwer), 399

Mardling, R. A., \& Aarseth, S. J. 2001, MNRAS, 321, 398

Mathieu, R. D., Latham, D. W., \& Griffin, R. F. 1990, AJ, 100, 1859

Mikkola, S. 1983, MNRAS, 203, 1107

Mikkola, S., \& Aarseth, S. J. 1993, Celest. Mech. Dyn. Astron., 57, 439

Raboud, R., \& Mermilliod, J.-C. 1998, A\&A, 329, 101

Saslaw, W. C., Valtonen, M. J., \& Aarseth, S. J. 1974, ApJ, 190, 253

Sterzik, M. F., \& Tokovinin, A. A. 2002, A\&A, 384, 1030.

Tout, C. A., Aarseth, S. J., Pols, O. R., \& Eggleton, P. P. 1997, MNRAS, 291, 732

van Leeuwen, F., \& van Genderen, A. M. 1997, A\&A, 327,1070

Zare, K. 1977, Celest. Mech., 16, 35

Sverre J. Aarseth: Institute of Astronomy, University of Cambridge, Madingley Road, Cambridge CB3 0HA, UK (sverre@ast.cam.ac.uk). 


\section{DISCUSSION}

Sterzik - Is the fraction of short-period binaries you find in the dynamical simulations consistent with observations?

Aarseth - The initial distribution of periods is flat in $\log (\mathrm{a})$, in accord with observations.

Zinnecker - The maximum primordial binary fraction you used in your star-cluster simulations was $20 \%$. However, observations suggest that this fraction can easily be as high as $50 \%$ (e.g., in the Orion Trapezium cluster). What would be the effect of increasing your primordial binary fraction?

Aarseth - All the binaries used in the simulations are hard. Hence neglecting an even larger fraction of soft binaries would not have much effect because of their small combined binding energy.

Milone - You have included so many aspects of the environment in your simulations that I am wondering if you have also considered including the effects of varying tidal effects due to a globular cluster's varying distance from the galactic centre.

Aarseth - The cluster model discussed here is for an open cluster in a circular orbit. For globular star simulations which we are about to start, the cluster orbit will be at varying distances from the Galactic centre. Also, tidal shocks from passages through the Galactic plane will be included.

Mathieu - What percentage of the M67 population would you expect to be dynamically-formed hierarchical systems?

Aarseth - About 1\%.

$M$. Valtonen - Is the loss of binaries in the cluster mostly due to ejections? And is the ejection rate anywhere close to linear with time?

Aarseth - The majority of binaries escape from the cluster with their primordial membership. The escape rate of binaries is linear until half have been lost, then it starts to increase a bit.

Udry - Are you seeing a dependence of your multiple systems population on the distance to the cluster center?

Aarseth - The hierarchical systems tend to be centrally concentrated.

Udry - Masses are taken from an IMF. Is there a mass difference observed within a given category of your multiples, quadruples for example?

Aarseth - There is tendency for quadruples to be relatively massive, but the statistical sample is rather small. Likewise, high-order systems survive longer if they are massive.

Clarke - Could you quantify for the sake of observers the optimum age at which they should expect to find hierarchical systems in clusters? (Preferably a formula that could be scaled to clusters of different $\mathrm{N}$ and radius).

Aarseth - If we begin with no hierarchies, the maximum is reached near the half-life of the cluster. However, one might expect a primordial population, in which case the maximum could be reached during the early stages. 